\title{
Composites Europe vergrößert
}

Liebe Leserin, lieber Leser,

W enn Sie für kommenden Monat in Stuttgart noch kein Zimmer gebucht haben, könnte es knapp werden. Immerhin erwartet die Composites Europe vom 22. bis 24 . September mehr als 11.000 Besucher, und da gilt die bekannte Weisheit mit dem frühen Vogel...

Wie das Interesse auch an dieser Veranstaltung zeigt, ist Leichtbau kein vergängliches Modewort. Die werkstoffübergreifende Disziplin beschäftigt mittlerweile ganze Stäbe von Forschern, Konstrukteuren oder Experten der Produktionstechnik weltweit. Viele neue Erkenntnisse und Praxiserfahrungen sind zwangsläufiges Ergebnis dieser Aktivität und füllen Säle einschlägiger Foren und Präsentationen. Auch die Composites Europe meldete bereits im Juni eine höhere Nachfrage nach Ausstellungsfläche und stockt in diesem Jahr um eine weitere Halle auf, um den rund 450 internationalen Ausstellern genügend Raum für ihre Exponate zu bieten.

Neu ist der von der Wirtschaftsvereinigung Composites Germany durchgeführte erste International Composite Congress, der die internationale AVK-Tagung ersetzt und am 21. und 22. September die Auftaktveranstaltung bildet. Hier tauschen Experten aus ganz Europa ihre Erkenntnisse aus.

Neben der klassischen "Composites Europe" sollen in diesem Jahr bei der Hybrid Expo wieder diejenigen auf ihre Kosten kommen, die sich nicht nur mit sehr speziellen Fragestellungen, sondern mit der gesamten Prozesskette zur seriellen Fertigung hybrider Bauteile befassen. Hauptadressat ist die Automobilindustrie auch bei der neuen Konferenz bio!CAR, die Informationen rund um das Thema biobasierte, nachwachsende Materialien bereithält und die ergänzend mit einem Themenpavillon Produkte zeigt, die sich schon heute aus Naturfasern herstellen lassen. Ebenso gibt es mit der zweiten "Industry meets Science" eine Sonderfläche, bei der unter Federführung des Instituts für Kunststoffverarbeitung (IKV) der RWTH Aachen neue Forschungsergebnisse, zum Beispiel Methoden zur Schadensanalyse und werkstoffgerechten Reparatur, nähergebracht werden.

Die letzte Großveranstaltung in diesem Jahr zeigt mit ihrer stetigen Entwicklung, dass die Bemühungen im Leichtbau mit faserverstärkten Kunststoffen zu immer neuen Erkenntnissen führt. Diese Erfahrungen auf Nutzwert, Machbarkeit und Sinnhaftigkeit zu diskutieren, bleibt permanente Aufgabe. So bleibt zu wünschen, dass auch die diesjährige Messe wieder zum „Aha-Effekt" beiträgt und der Branche weiter Auftrieb gibt.

Ihr

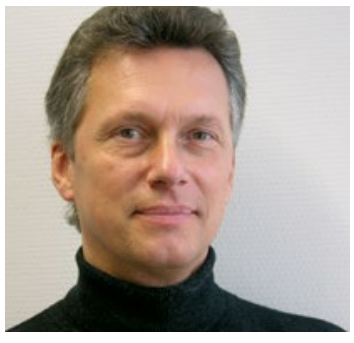

Dipl.-Ing. Ulrich Knorra Redakteur ulrich.knorra@springer.com 